\title{
Calciphylaxis epidemiology, risk factors, treatment and survival among French chronic kidney disease patients: a case- control study
}

Raphaël Gaisne ${ }^{1,2^{*}}$ D, Morgane Péré ${ }^{3}$, Victorio Menoyo ${ }^{4}$, Maryvonne Hourmant ${ }^{1}$ and David Larmet-Burgeot ${ }^{1,5}$

\begin{abstract}
Background: Calcific Uremic Arteriolopathy (CUA) is a rare disease, causing painful skin ulcers in patients with end stage renal disease. Recommendations for CUA management and treatment are lacking.

Methods: We conducted a retrospective cohort study on CUA cases identified in western France, in order to describe its management and outcome in average clinical practices. Selection was based on the Hayashi diagnosis criteria (2013) extended to patients with eGFR $<30 \mathrm{~mL} / \mathrm{min} / 1.73 \mathrm{~m}^{2}$. Dialyzed CUA cases were compared with 2 controls, matched for age, gender, region of treatment and time period.

Results: Eighty-nine CUA cases were identified between 2006 and 2016, including 19 non dialyzed and 70 dialyzed patients. Females with obesity (55.1\%) were predominant. Bone mineral disease abnormalities, inflammation and malnutrition (weight loss, serum albumin decrease) preceded CUA onset for 6 months. The multimodal treatment strategy included wound care (98.9\%), antibiotherapy (77.5\%), discontinuation of Vitamin K antagonists (VKA) (70.8\%) and intravenous sodium thiosulfate (65.2\%). 40.4\% of the patients died within the year after lesion onset, mainly under palliative care. Surgical debridement, distal CUA, localization to the lower limbs and non calciumbased phosphate binders were associated with better survival. Risks factors of developing CUA among dialysis patients were obesity, VKA, weight loss, serum albumin decrease or high serum phosphate in the 6 months before lesion onset.
\end{abstract}

Conclusion: CUA involved mainly obese patients under VKA. Malnutrition and inflammation preceded the onset of skin lesions and could be warning signs among dialysis patients at risk.

Trial registration: ClinicalTrials.gov identifier NCT02854046, registered August 3, 2016.

Keywords: Calcific uremic arteriolopathy, Calciphylaxis, Case-control study, ESRD, Vitamin K antagonist

\section{Background}

Calcific uremic arteriolopathy (CUA), also called calciphylaxis, is a rare but devastating disease involving patients with end stage renal disease (ESRD). CUA causes painful skin lesions that evolve to ulcerative lesions at risk of superinfection and sepsis [1], with a poor

\footnotetext{
* Correspondence: raphael.gaisne@chu-nantes.fr

'Department of Nephrology and Immunology, Institute of Transplantation Urology and Nephrology, Centre Hospitalier Universitaire de Nantes, Nantes, France

${ }^{2}$ Service de Néphrologie et Immunologie Clinique, Centre Hospitalier Universitaire de Nantes, 30, bd Jean Monnet 44093, Nantes Cedex 01, France Full list of author information is available at the end of the article
}

prognosis. One year survival rates vary between 45 to $55 \%$ [2-4]. CUA management lacks strong recommendations [5] and therefore is heterogeneous. Reported risk factors of CUA are female sex, obesity, diabetes mellitus, vitamin K antagonists (VKA) and ESRD [6]. Dysregulation of calcium-phosphate metabolism also participates to its development. Histopathological findings of skin lesions mostly associate thromboses and vessel calcifications [7]. We decided to conduct the first study on CUA in the French population. Our main objective was to describe diagnosis management, treatment and outcome of CUA in ESRD and stage 4-5 CKD patients. Secondary

(C) The Author(s). 2020 Open Access This article is distributed under the terms of the Creative Commons Attribution 4.0 International License (http://creativecommons.org/licenses/by/4.0/), which permits unrestricted use, distribution, and 
objectives were to analyze risk factors of developing calciphylaxis and influencing patient survival in the dialyzed cohort.

\section{Materials and methods Study patients}

We first conducted a retrospective cohort study and secondly a case control analysis. The nephrologists from Western France were asked to report their patients diagnosed for CUA. Patients were also identified by searching the MEDIAL dialysis regional data base. Inclusion and non-inclusion criteria checking and collection of the data in the medical records were performed by the investigators in the center of care of each case.

Hayashi [8] criteria were used for diagnosis: chronic hemodialysis or estimated Glomerular Filtration Rate (eGFR) below $15 \mathrm{~mL} / \mathrm{min} / 1.73 \mathrm{~m}^{2}$, more than two painful non-treatable skin ulcers with concomitant painful purpura and localization of skin ulcers on the trunk, extremities or penis with concomitant painful purpura. Typical histopathological findings (necrosis and ulceration of the skin with calcification of the tunica media and internal elastic membrane of small to medium-sized arterioles of dermis and subcutaneous fat) can replace a clinical feature.

Inclusion criteria were: CUA according to Hayashi criteria, onset of cutaneous lesions between 1st January 2006 and 31th December 2016, patients $>18$ yo. Patients with eGFR between 15 and $30 \mathrm{~mL} / \mathrm{min} / 1.73 \mathrm{~m}^{2}$ (CKD EPI formula) (the serum creatinine at onset of CUA was considered) were also included if all other inclusion criteria were met. CUA was eliminated if a differential diagnosis seemed more likely or was confirmed by skin biopsy, or in case of severe atherosclerotic vascular disease in the wound area. CUA patients under hemodialysis or peritoneal dialysis at onset of CUA lesions were assigned to the dialysis group, while the others to the non dialysis group.

\section{Controls selection}

In order to explore risk factors of CUA among dialyzed patients, each CUA dialysis patient was matched with two controls identified in the REIN registry of treated ESRD in France. Matching criteria were: gender, age ( \pm 2 years), treatment by hemodialysis in the same geographical area and at the same time of CUA diagnosis in the case. Among the potential controls for each case, two were randomly and anonymously selected. After checking the absence of diagnosis of CUA, collection of the data in the medical records of the control patients was performed by the investigator directly in the center of care of the patient.

\section{Study data}

The analyzed data were demographic, history of kidney disease, cardiovascular comorbidities and other previously reported risk factors of CUA. Thrombophilia was defined as acquired or congenital antithrombin/protein $\mathrm{C} /$ protein $\mathrm{S}$ deficiency, antiphospholipid syndrome, activated protein $\mathrm{C}$ resistance, prothrombin mutation. "Onset date" of CUA was the date where typical skin lesions were mentioned in medical records. Laboratory data at onset were recorded as well as the worst values within the 6 months before diagnosis. As intact Parathyroid hormone (iPTH) measurement kits were different between laboratories, we normalized iPTH with the upper limit of the normal range for each laboratory. Medications and dialysis parameters were recorded. For each CUA case, clinical presentation with lesion distribution defined as proximal (extremities proximal to knees and elbows, trunk, breast and penis) and/or distal (extremities distal to knees and elbows), evolution, diagnosis and treatment methods and outcomes were collected.

A written consent form was given to each patient, except for deceased patients and loss of follow-up patients. The study was approved by the ethics committee of the Nantes University Hospital. All data collected were de-identified.

\section{Statistical analyses}

Frequency of categorical variables, median and interquartile range (IQR) for non-normally distributed variables were reported. Survival curves were determined using the Kaplan-Meier method. Survival analysis using Cox models were used to determine survival predictors. In the group of CUA patients treated by dialysis, univariate conditional logistic regression analysis on matched case-controls was performed. Variables with $p<0.20$ were included in the multivariate logistic regression analysis. Non dialyzed CUA patients were not included in the risk factor analysis because of the lack of controls for those patients.

All analyses were performed using the SAS program (version 9.4) (SAS Institute Inc., Cary, NC). Statistical

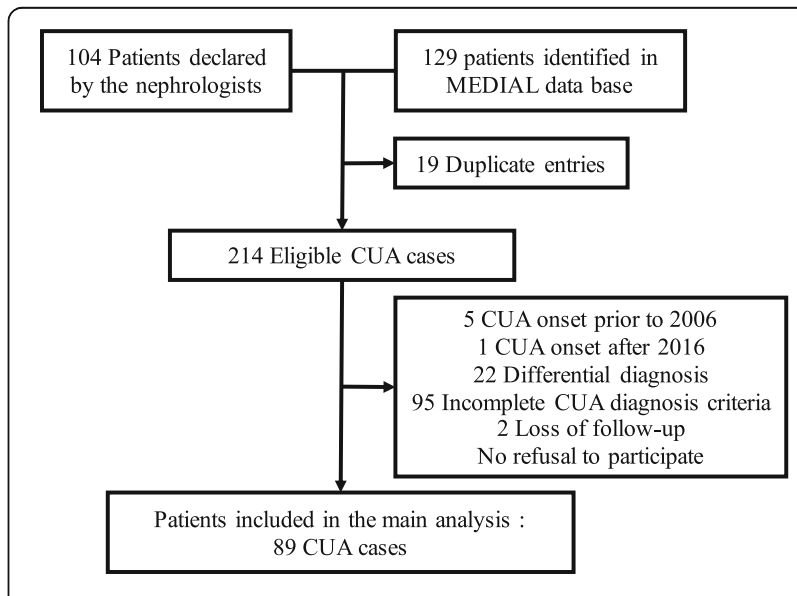

Fig. 1 Flow chart of selection of Calcific Uremic Arteriolopathy cases 
significance was set as $p<0.05$. The study protocol was pre-registered on clinicaltrials.gov under the number NCT02854046.

\section{Results}

Two hundred fourteen eligible cases were identified (Fig. 1). Ninety-five patients with incomplete diagnosis criteria and 22 with a differential diagnosis were excluded (Table S1). Eighty-nine cases of CUA were finally included, $74 \%$ diagnosed between 2013 and 2016.
Baseline characteristics (Table 1)

$75.3 \%$ of cases were hemodialyzed. Among the 19 patients with stage $4-5$ chronic kidney disease (CKD), median eGFR was $10.3 \mathrm{ml} / \mathrm{min} / 1.73 \mathrm{~m}^{2}$ (IQR $8.4-13.0$ ) and median blood urea nitrogen was $31 \mathrm{mmol} / \mathrm{L}$ (IQR 2047). CUA patients were obese (median Body Mass Index (BMI) $31 \mathrm{~kg} / \mathrm{m}^{2}$ ) and had a recent median weight loss of $3.5 \mathrm{~kg}$. In dialyzed and non dialyzed CUA cases, the main causes of CKD were respectively diabetesassociated nephropathy (25.7 and $26.3 \%)$, hypertensionassociated nephropathy (22.9 and 0\%), hypertension and

Table 1 Demographic data of CUA cases and the matched dialysis controls

\begin{tabular}{|c|c|c|c|c|c|c|c|c|c|}
\hline Parameter & $\mathrm{N}$ & Total CUA cases & $N$ & Non dialysis cases & $\mathrm{N}$ & Dialysis cases & $\mathrm{N}$ & Dialysis controls & $P$ Value \\
\hline Age (years) & 89 & $70(63-78)$ & 19 & $71(58-79)$ & 70 & $70(64-78)$ & 140 & $69(63-77)$ & \\
\hline Females & 89 & $57(64.0 \%)$ & 19 & $8(42.1 \%)$ & 70 & $49(70.0 \%)$ & 140 & $98(70.0 \%)$ & \\
\hline BMI $\left(\mathrm{kg} / \mathrm{m}^{2}\right)$ & 89 & $31.0(25.3-37.3)$ & 19 & $34.6(28.6-39.2)$ & 70 & $30.7(24.5-37.1)$ & 137 & $25.1(21.6-28.6)$ & $<0.001$ \\
\hline $\begin{array}{l}\text { Overweight } \\
\text { (BMl 25-30 kg/m2) }\end{array}$ & & $20(22.5 \%)$ & & $6(31.6 \%)$ & & $14(20.0 \%)$ & & $40(29.2 \%)$ & \\
\hline $\begin{array}{l}\text { Obesity } \\
\text { (BMl } 30-40 \text { kg/m2) }\end{array}$ & & $33(37.1 \%)$ & & $10(52.6 \%)$ & & $23(32.9 \%)$ & & $26(19.0 \%)$ & \\
\hline $\begin{array}{l}\text { Severe obesity } \\
(\mathrm{BMl}>40 \mathrm{~kg} / \mathrm{m} 2)\end{array}$ & & $16(18.0 \%)$ & & $3(15.8 \%)$ & & $13(18.6 \%)$ & & $3(2.2 \%)$ & \\
\hline $\begin{array}{l}\text { Loss of weight within } \\
6 \text { months before } \\
\text { diagnosis }(\mathrm{kg})\end{array}$ & 82 & $3.5(0.5-7.0)$ & 16 & $7.0(3.0-15.9)$ & 66 & $3.0(0.0-6.0)$ & 134 & $0.0(-1.1-1.5)$ & $<0.001$ \\
\hline CKD stage (eGFR) & 89 & & 19 & & 70 & & 140 & & \\
\hline $\begin{array}{l}\text { CKD Stage } 4 \\
\left(15-30 \mathrm{~mL} / \mathrm{min} / 1.73 \mathrm{~m}^{2}\right)\end{array}$ & & $4(4.5 \%)$ & & $4(21.0 \%)$ & & & & & \\
\hline $\begin{array}{l}\text { CKD Stage } 5 \\
\left(<15 \mathrm{~mL} / \mathrm{min} / 1.73 \mathrm{~m}^{2}\right)\end{array}$ & & $15(16.8 \%)$ & & $15(70.0 \%)$ & & & & & \\
\hline $\begin{array}{l}\text { CKD stage } 5 \text { under } \\
\text { Hemodialysis }\end{array}$ & & $67(75.3 \%)$ & & & & 67 (95.7\%) & & 140 (100\%) & \\
\hline CKD stage 5 under PD & & $3(3.4 \%)$ & & & & $3(4.2 \%)$ & & & \\
\hline CAD & 89 & $41(46.1 \%)$ & 19 & $6(31.6 \%)$ & 70 & $35(50.0 \%)$ & 140 & $50(35.7 \%)$ & 0.047 \\
\hline Heart failure & 89 & $51(57.3 \%)$ & 19 & $11(57.9 \%)$ & 70 & $40(57.1 \%)$ & 140 & $34(24.3 \%)$ & $<0.001$ \\
\hline Stroke & 89 & $14(15.7 \%)$ & 19 & $3(15.8 \%)$ & 70 & $11(15.7 \%)$ & 140 & $25(17.9 \%)$ & 0.70 \\
\hline PAD with symptoms & 89 & 35 (39.3\%) & 19 & $4(21.1 \%)$ & 70 & $31(44.3 \%)$ & 140 & $40(28.6 \%)$ & 0.02 \\
\hline Diabetes mellitus & 89 & $60(67.4 \%)$ & 19 & 17 (89.5\%) & 70 & $43(61.4 \%)$ & 140 & $56(40.0 \%)$ & 0.003 \\
\hline Arterial Hypertension & 89 & 85 (95.5\%) & 19 & $18(94.7 \%)$ & 70 & 67 (95.7\%) & 140 & $121(86.4 \%)$ & 0.04 \\
\hline Hypercholesterolemia & 89 & $52(58.4 \%)$ & 19 & 11 (57.9\%) & 70 & $41(58.6 \%)$ & 140 & $87(62.1 \%)$ & 0.61 \\
\hline History of smoking & 89 & $20(22.5 \%)$ & 19 & $6(31.6 \%)$ & 70 & $14(20.0 \%)$ & 137 & $23(16.8 \%)$ & 0.57 \\
\hline Parathyroidectomy & 89 & $4(4.5 \%)$ & 19 & $0(0.0 \%)$ & 70 & $4(5.7 \%)$ & 140 & $8(5.71 \%)$ & 1 \\
\hline $\begin{array}{l}\text { History of pathologic } \\
\text { fracture }\end{array}$ & 89 & $19(21.3 \%)$ & 19 & $2(10.5 \%)$ & 70 & $17(24.3 \%)$ & 140 & $17(12.1 \%)$ & 0.02 \\
\hline Progressive cancer & 89 & $9(10.1 \%)$ & 19 & $2(10.5 \%)$ & 70 & $7(10.0 \%)$ & 140 & $14(10.0 \%)$ & 1 \\
\hline Hepatobiliary disease & 89 & $15(16.9 \%)$ & 19 & $2(10.5 \%)$ & 70 & $13(18.6 \%)$ & 140 & $16(11.4 \%)$ & 0.16 \\
\hline Chronic alcoholism & 89 & 7 (7.9\%) & 19 & $2(10.5 \%)$ & 70 & $5(7.1 \%)$ & 140 & $6(4.3 \%)$ & 0.51 \\
\hline Connective tissue disease & 89 & $7(7.9 \%)$ & 19 & $2(10.5 \%)$ & 70 & $5(7.1 \%)$ & 140 & $5(3.6 \%)$ & 0.31 \\
\hline Thrombophilia & 89 & $5(5.6 \%)$ & 19 & $0(0.0 \%)$ & 70 & $5(7.1 \%)$ & 140 & $8(5.7 \%)$ & 0.76 \\
\hline
\end{tabular}

Median (IQR) or N (\%). P-Value of comparison of Dialysis Cases with Dialysis controls. ADPKD autosomal dominant polycystic kidney disease, $B M I$ body mass index, $C A D$ coronary artery disease, $C K D$ chronic kidney disease, CUA calcific uremic arteriolopathy, PAD peripheral artery disease, $P D$ peritoneal dialysis 
diabetes-associated nephropathy (15.7 and $21.1 \%$ ) and glomerular nephropathy (10.0 and 26.3\%). 5 CUA patients only had proven thrombophilia.

\section{Laboratory findings}

Adjusted serum calcium, serum phosphate and normalized $\mathrm{iPTH}$ were significantly higher in dialyzed CUA patients than in matched dialyzed controls at lesion onset and in the six preceding months (Table 2). Malnutrition preceded CUA onset, with a median albumin decrease of $2.7 \mathrm{~g} / \mathrm{L}$ within the 6 months before onset and Creactive protein (CRP) was high at both times.

\section{Medications at CUA lesion onset}

Active and native vitamin D were not significantly more prescribed in CUA patients, nor statin and cinacalcet (Table 3). Calcium-based phosphate binders were more frequently administered in CUA patients; $71.9 \%$ were under VKA. The median time between VKA introduction and onset of CUA was 3.2 years (IQR 1.8-6.6) and was shorter for dialyzed vs non dialyzed patients $(2.6$

Table 2 Laboratory parameters measured at onset of CUA and within 6 months before diagnosis (most pejorative value) in CUA and paired dialysis controls

\begin{tabular}{|c|c|c|c|c|c|c|c|c|c|c|}
\hline Biological parameter & $\begin{array}{l}\text { Recommended } \\
\text { range }^{\mathrm{a}}\end{array}$ & $\mathrm{N}$ & $\begin{array}{l}\text { Total CUA } \\
\text { cases }\end{array}$ & $\mathrm{N}$ & $\begin{array}{l}\text { Non dialysis } \\
\text { cases }\end{array}$ & $N$ & $\begin{array}{l}\text { Dialysis } \\
\text { cases }\end{array}$ & $\mathrm{N}$ & $\begin{array}{l}\text { Dialysis } \\
\text { controls }\end{array}$ & $\begin{array}{l}P \\
\text { Value }\end{array}$ \\
\hline \multicolumn{11}{|l|}{ At onset of lesions } \\
\hline Total serum calcium (mmol/L) & $2.10-2.60$ & 89 & $\begin{array}{l}2.25(2.12- \\
2.35)\end{array}$ & 19 & $\begin{array}{l}2.25(2.20- \\
2.34)\end{array}$ & 70 & $\begin{array}{l}2.23(2.11- \\
2.38)\end{array}$ & 138 & $\begin{array}{l}2.18(2.08- \\
2.30)\end{array}$ & 0.04 \\
\hline Adjusted serum calcium (mmol/L) & $2.10-2.60$ & 88 & $\begin{array}{l}2.50(2.33- \\
2.60)\end{array}$ & 18 & $\begin{array}{l}2.51(2.43- \\
2.68)\end{array}$ & 70 & $\begin{array}{l}2.48(2.31- \\
2.58)\end{array}$ & 136 & $\begin{array}{l}2.26(2.16- \\
2.40)\end{array}$ & $\begin{array}{l}< \\
0.001\end{array}$ \\
\hline Serum phosphate (mmol/L) & $0.8-1.5$ & 89 & $\begin{array}{l}1.89(1.50- \\
2.34)\end{array}$ & 19 & $\begin{array}{l}1.98(1.64- \\
2.60)\end{array}$ & 70 & $\begin{array}{l}1.87(1.46- \\
2.29)\end{array}$ & 138 & $\begin{array}{l}1.42(1.13- \\
1.87)\end{array}$ & $\begin{array}{l}< \\
0.001\end{array}$ \\
\hline Calcium phosphate product $\left(\mathrm{mmol}^{2} / \mathrm{L}^{2}\right)$ & & 88 & $\begin{array}{l}4.35(3.29- \\
5.23)\end{array}$ & 18 & $\begin{array}{l}4.59(3.70- \\
5.74)\end{array}$ & 70 & $\begin{array}{l}4.21(3.19- \\
5.22)\end{array}$ & 138 & $\begin{array}{l}3.18(2.50- \\
4.01)\end{array}$ & $\begin{array}{l}< \\
0.001\end{array}$ \\
\hline iPTH (pg/mL) & $150-600$ & 87 & $\begin{array}{l}260(114- \\
605)\end{array}$ & 17 & $115(83-488)$ & 70 & $\begin{array}{l}336(141- \\
605)\end{array}$ & 136 & $\begin{array}{l}272(157- \\
466)\end{array}$ & 0.20 \\
\hline Normalized iPTH (N) & $2-9$ & 87 & $5.3(2.1-11.1)$ & 17 & $2.2(1.6-10.6)$ & 70 & $\begin{array}{l}5.3(2.5- \\
11.1)\end{array}$ & 136 & $4.4(2.4-8.4)$ & 0.04 \\
\hline $\begin{array}{l}\text { iPTH outside of target range between } 2 \text { and } 9 \text { fold } \\
\text { normal range }\end{array}$ & & 87 & $51(58.6 \%)$ & 17 & $14(73.7 \%)$ & 70 & $37(52.9 \%)$ & 136 & $58(42.6 \%)$ & 0.16 \\
\hline 25-Hydroxyvitamin D (ng/mL) & $>30$ & 68 & $\begin{array}{l}28.5(17.9- \\
40.0)\end{array}$ & 13 & $\begin{array}{l}24.0(10.0- \\
34.0)\end{array}$ & 55 & $\begin{array}{l}29.9(18.0- \\
40.0)\end{array}$ & 101 & $\begin{array}{l}34.1(22.0- \\
47.6)\end{array}$ & 0.18 \\
\hline Serum Albumin (g/L) & $35-45$ & 88 & $\begin{array}{l}30.9(28.0- \\
34.0)\end{array}$ & 18 & $\begin{array}{l}30.4(23.9- \\
34.3)\end{array}$ & 70 & $\begin{array}{l}31.5(28.0- \\
34.0)\end{array}$ & 136 & $\begin{array}{l}37.0(33.3- \\
39.2)\end{array}$ & $<0.01$ \\
\hline $\begin{array}{l}\text { Serum Albumin variation between diagnosis and } 6 \\
\text { months before }(\mathrm{g} / \mathrm{L})\end{array}$ & & 80 & $\begin{array}{l}-2.7(-5.0 \\
1.6)\end{array}$ & 12 & $\begin{array}{l}-3.8(-8.5 \\
-0.5)\end{array}$ & 68 & $-2.7(-5.0$ & 134 & $1.3(0.0 ; 4.0)$ & $\begin{array}{l}< \\
0.001\end{array}$ \\
\hline $\mathrm{CRP}(\mathrm{mg} / \mathrm{L})$ & $<5$ & 87 & $\begin{array}{l}29.0(8.0- \\
72.0)\end{array}$ & 17 & $\begin{array}{l}34.0(17.5- \\
72.0)\end{array}$ & 70 & $\begin{array}{l}23.5(6.6- \\
64.0)\end{array}$ & 135 & $4.2(1.0-13.8)$ & $<.001$ \\
\hline Hemoglobin (g/dL) & $10-11.5$ & 89 & $\begin{array}{l}10.4(9.7- \\
11.6)\end{array}$ & 19 & $10.1(9.7-11.7)$ & 70 & $\begin{array}{l}10.6(9.6- \\
11.5)\end{array}$ & 136 & $\begin{array}{l}11.2(10.1- \\
12.0)\end{array}$ & 0.007 \\
\hline \multicolumn{11}{|l|}{ Worst value within 6 months before onset of CUA } \\
\hline Total serum calcium (mmol/L) & $2.10-2.60$ & 86 & $\begin{array}{l}2.34(2.17- \\
2.47)\end{array}$ & 17 & $\begin{array}{l}2.33(2.29- \\
2.38)\end{array}$ & 69 & $\begin{array}{l}2.35(2.17- \\
2.48)\end{array}$ & 137 & $\begin{array}{l}2.28(2.20- \\
2.37)\end{array}$ & 0.30 \\
\hline Adjusted serum calcium (mmol/L) & $2.10-2.60$ & 81 & $\begin{array}{l}2.54(2.37- \\
2.68)\end{array}$ & 13 & $\begin{array}{l}2.54(2.41- \\
2.71)\end{array}$ & 68 & $\begin{array}{l}2.54(2.30- \\
2.67)\end{array}$ & 135 & $\begin{array}{l}2.43(2.31- \\
2.52)\end{array}$ & 0.05 \\
\hline Serum phosphorus (mmol/L) & $0.8-1.5$ & 86 & $\begin{array}{l}2.16(1.75- \\
2.57)\end{array}$ & 17 & $\begin{array}{l}2.00(1.55- \\
2.11)\end{array}$ & 69 & $\begin{array}{l}2.25(1.87- \\
2.70)\end{array}$ & 137 & $\begin{array}{l}1.72(1.44- \\
2.15)\end{array}$ & $\begin{array}{l}< \\
0.001\end{array}$ \\
\hline Calcium phosphate product $\left(\mathrm{mmol}^{2} / \mathrm{L}^{2}\right)$ & & 86 & $\begin{array}{l}4.81(4.24- \\
6.02)\end{array}$ & 17 & $\begin{array}{l}4.30(3.57- \\
4.82)\end{array}$ & 69 & $\begin{array}{l}5.20(4.44- \\
6.50)\end{array}$ & 137 & $\begin{array}{l}3.94(3.26- \\
4.88)\end{array}$ & $\begin{array}{l}< \\
0.001\end{array}$ \\
\hline iPTH (pg/mL) & $150-600$ & 75 & $\begin{array}{l}355(148- \\
710)\end{array}$ & 10 & $331(210-580)$ & 65 & $\begin{array}{l}435(148- \\
710)\end{array}$ & 128 & $\begin{array}{l}342(173- \\
526)\end{array}$ & 0.10 \\
\hline Normalized iPTH (N) & $2-9$ & 75 & $7.3(3.3-12.2)$ & 10 & $6.5(4.5-12.1)$ & 65 & $\begin{array}{l}7.3(3.3- \\
12.2)\end{array}$ & 128 & $5.3(2.8-8.5)$ & 0.02 \\
\hline $\begin{array}{l}\text { iPTH outside of target value between } 2 \text { and } 9 \text { fold normal } \\
\text { range }\end{array}$ & & 75 & $56(62.9 \%)$ & 10 & $15(79.0 \%)$ & 65 & $41(63.1 \%)$ & 128 & $59(46.1 \%)$ & 0.01 \\
\hline 25-Hydroxyvitamin D (ng/mL) & $>30$ & 59 & $\begin{array}{l}29.0(15.0- \\
42.8)\end{array}$ & 9 & $13.0(9.0-25.0)$ & 50 & $\begin{array}{l}30.0(18.0- \\
43.0)\end{array}$ & 101 & $\begin{array}{l}36.1(20.4- \\
48.0)\end{array}$ & 0.32 \\
\hline Serum Albumin (g/L) & $35-45$ & 81 & $\begin{array}{l}33.0(29.0- \\
37.0)\end{array}$ & 13 & $\begin{array}{l}32.9(25.0- \\
37.5)\end{array}$ & 68 & $\begin{array}{l}33.5(29.0- \\
37.0)\end{array}$ & 135 & $\begin{array}{l}34.0(32.0- \\
38.0)\end{array}$ & 0.049 \\
\hline CRP (mg/L) & $<5$ & 80 & $\begin{array}{l}39.5(14.0- \\
79.6)\end{array}$ & 13 & $32.0(5.9-56.0)$ & 67 & $\begin{array}{l}46.0(14.1- \\
79.9)\end{array}$ & 133 & $\begin{array}{l}13.4(4.5- \\
40.2)\end{array}$ & 0.004 \\
\hline
\end{tabular}

Median (IOR) or N (\%). P-Value of comparison between dialysis cases and dialysis controls. CRP, C-reactive protein; iPTH, intact parathyroid hormone ${ }^{a}$ According to KDIGO clinical practice guideline for the diagnosis, evaluation, prevention, and treatment of chronic kidney disease-mineral and bone disorder (CKD-MBD) Kidney Int Suppl 2009; 113: S1-S130. Adjusted serum calcium level was calculated using the following formula: [serum calcium (mmol/L) + 0,025 (40-Albumin)] 
Table 3 Medications at time of diagnosis of CUA in cases and matched dialysis controls

\begin{tabular}{|c|c|c|c|c|c|c|c|c|c|}
\hline Treatment & $\mathrm{N}$ & Total CUA cases & $\mathrm{N}$ & Non dialysis cases & $\mathrm{N}$ & Dialysis cases & $\mathrm{N}$ & Dialysis controls & $P$ Value \\
\hline 25-hydroxyvitamin D & 89 & $53(59.6 \%)$ & 19 & $11(57.9 \%)$ & 70 & $42(60.0 \%)$ & 137 & $89(65.0 \%)$ & 0.48 \\
\hline Active vitamin D & 89 & $19(21.4 \%)$ & 19 & $3(15.8 \%)$ & 70 & $16(22.9 \%)$ & 137 & $31(22.6 \%)$ & 0.97 \\
\hline Calcium-based phosphate binders & 89 & $47(52.8 \%)$ & 19 & $6(31.6 \%)$ & 70 & $41(58.6 \%)$ & 137 & $60(43.8 \%)$ & 0.04 \\
\hline Non calcium-based phosphate binders & 89 & $47(52.8 \%)$ & 19 & $4(21.1 \%)$ & 70 & $43(61.4 \%)$ & 137 & $76(55.6 \%)$ & 0.41 \\
\hline Sevelamer & 89 & $37(41.6 \%)$ & 19 & $4(21.1 \%)$ & 70 & $33(47.1 \%)$ & 137 & $58(41.4 \%)$ & 0.43 \\
\hline Lanthanum carbonate & 89 & $12(13.5 \%)$ & 19 & $1(5.3 \%)$ & 70 & $11(15.7 \%)$ & 137 & $19(13.6 \%)$ & 0.68 \\
\hline Cinacalcet & 89 & $17(19.1 \%)$ & 19 & $1(5.3 \%)$ & 70 & $16(22.9 \%)$ & 136 & $26(19.1 \%)$ & 0.53 \\
\hline Betablocker & 89 & $54(60.7 \%)$ & 19 & $15(79.0 \%)$ & 70 & $39(55.7 \%)$ & 137 & 65 (47.5\%) & 0.26 \\
\hline Insulin therapy & 89 & 35 (39.3\%) & 19 & $8(42.1 \%)$ & 70 & $27(38.6 \%)$ & 138 & $34(24.6 \%)$ & 0.04 \\
\hline Vitamin K Antagonist & 89 & $64(71.9 \%)$ & 19 & $11(57.9 \%)$ & 70 & $53(75.7 \%)$ & 138 & $37(26.8 \%)$ & $<0.001$ \\
\hline Fluindione & 64 & $44(68.8 \%)$ & 11 & $6(54.6 \%)$ & 53 & $38(71.7 \%)$ & 37 & $18(48.7 \%)$ & \\
\hline Warfarin & 64 & $17(26.6 \%)$ & 11 & $3(27.7 \%)$ & 53 & $14(26.4 \%)$ & 37 & $16(43.2 \%)$ & \\
\hline Corticosteroids & 89 & $10(11.2 \%)$ & 19 & $2(10.5 \%)$ & 70 & $8(11.4 \%)$ & 138 & $16(11.6 \%)$ & 0.97 \\
\hline Statin & 89 & $46(51.7 \%)$ & 19 & $9(47.4 \%)$ & 70 & $37(52.7 \%)$ & 138 & $71(51.5 \%)$ & 0.85 \\
\hline ESA & 89 & $72(80.9 \%)$ & 19 & $9(47.4 \%)$ & 70 & $63(90.0 \%)$ & 137 & $106(77.4 \%)$ & 0.03 \\
\hline Iron therapy & 89 & $63(70.8 \%)$ & 19 & $8(42.1 \%)$ & 70 & $55(78.6 \%)$ & 137 & $102(74.4 \%)$ & 0.51 \\
\hline ACEi/ARB & 89 & $32(36.0 \%)$ & 19 & $8(42.1 \%)$ & 70 & $24(34.3 \%)$ & 138 & $43(31.2 \%)$ & 0.65 \\
\hline \multicolumn{10}{|l|}{ Hemodialysis parameters } \\
\hline $\mathrm{HD}$ & & & & & 67 & $43(64.2 \%)$ & 138 & 91 (65.9\%) & \\
\hline $\mathrm{HDF}$ & & & & & 67 & 47 (34.1\%) & 138 & $24(35.8 \%)$ & \\
\hline eKt/N & & & & & 62 & $1.40(1.06-1.67)$ & 131 & $1.53(1.33-1.82)$ & $<0.001$ \\
\hline Citrate & & & & & 67 & $10(14.3 \%)$ & 138 & $20(14.3 \%)$ & 1.00 \\
\hline
\end{tabular}

Median (IQR) or N (\%). P-Value of comparison of Dialysis Cases with Dialysis controls. ACEi/ARB angiotensin converting enzyme inhibitor/angiotensin receptor blocker; ESA erythropoiesis-stimulating agent, $H D$ hemodialysis, HDF hemodiafiltration

years (IQR 1.3-5.7) vs 6.5 years (IQR 4.6-8.6)). The median time between dialysis initiation and CUA was 1.4 years (IQR 0.3-3.7). Median dialysis vintage among the control patients, estimated between the beginning of dialysis and the time of onset of CUA in the matched case, was 2.2 years (IQR 0.7-5.1). Median dialysis dose (eKt/ V) was significantly lower for CUA cases compared to controls.

\section{Clinical presentation}

Fifty-nine CUA cases (66.2\%) had a triggering event within the 3 months before onset. Twenty-eight cases (31.5\%) had a local trauma, including physical trauma (21\%), subcutaneous injection of heparin $(25 \%)$ or insulin $(43 \%)$ or both (11\%). Thirty-five cases $(39.3 \%)$ had a hypovolemia episode, including sepsis (29\%), general anesthesia (11\%), severe intradialytic hypotension (11\%), acute heart failure $(11 \%)$, severe nephrotic syndrome (9\%), hemorrhage (5.7\%) and multifactorial causes (23\%). The same proportion of triggering event was found in dialyzed cases than in non-dialyzed cases (local trauma $30 \%$ vs $36.8 \%$, episode of hypovolemia $38.6 \%$ vs $42.1 \%$ respectively).
Thirty-six patients (40.5\%) suffered from a proximaltype CUA, while $26(29.2 \%)$ had a distal-type, and 27 (30.3\%) both proximal and distal. Lower limbs were involved in most of the patients $(86.5 \%)$, especially under the knees (34.8\%), while trunk lesions were found in $50.6 \%$, mainly in the abdomen (27.0\%). Upper limb lesions were present in $22.5 \%$. A median of 5 lesions (IQR $3-6)$ per patient were found and were mostly ulcerative (95.5\%).

\section{CUA diagnosis}

The median time between onset of skin lesions and diagnosis was 46 days (IQR 24-88). When standard X-rays were performed (57.3\%), calcifications were identified in arteries $(29.4 \%)$, arterioles $(15.7 \%)$ or both $(31.4 \%)$, or vessels with extravascular calcifications (17.6\%). In 24 patients $(27.0 \%)$ examined by CT-scan, calcifications were identified in $75 \%$ of them. 12 out of 18 patients (66.7\%) had a pathological nuclear bone scan. Transcutaneous oxygen measurement was pathological in 9 out of 11 evaluated patients. Doppler ultrasound (53 patients, $59.6 \%)$ revealed mostly medial calcification sclerosis associated with non-significant stenosis. 
A skin biopsy was performed in 60 patients $(67.4 \%)$, more frequently among non-dialyzed cases $(79.0 \%$ vs $64.3 \%)$, with an average number of $1.6( \pm 0.8)$ biopsies per patient, and confirmed the diagnosis in $65 \%$ of cases. A specialized calcium staining was performed for 33.3\% of cases, revealing calcification of arterioles (53.3\%), tissues $(8.3 \%)$, or both $(10 \%)$. The other findings were thrombosis (50\%), fibro-intimal hyperplasia $(20 \%)$ and panniculitis (72\%).

\section{CUA treatments}

Treatments used for CUA were wound care, intravenous Sodium Thiosulfate (STS), renal replacement therapy modification and nutritional support therapy (Table 4). Other treatments were discontinued, notably VKA, calcium supply and vitamin D. Median dosing of intravenous STS was $75 \mathrm{~g}$ per week, and the cumulative dose was higher for dialysis patients. Some treatments were scarcely used, and included, intra-lesional STS (1.1\%),

Table 4 Multimodal treatment strategy of CUA: initiation and modifications of treatments after CUA diagnosis

\begin{tabular}{|c|c|c|c|c|c|c|}
\hline Treatment & $\mathrm{N}$ & Total CUA cases & $\mathrm{N}$ & Dialysis cases & $\mathrm{N}$ & Non dialysis cases \\
\hline Wound care & 89 & 88 (98.9\%) & 70 & $70(100.0 \%)$ & 19 & $18(94.7 \%)$ \\
\hline Antibiotherapy & 89 & $69(77.5 \%)$ & 70 & $53(75.7 \%)$ & 19 & $16(84.2 \%)$ \\
\hline Discontinuation of VKA & 65 & $46(70.8 \%)$ & 54 & $38(70.4 \%)$ & 11 & $8(72.7 \%)$ \\
\hline Discontinuation of active vitamin $\mathrm{D}$ & 20 & $14(70.0 \%)$ & 17 & $11(64.7 \%)$ & 3 & $3(100.0 \%)$ \\
\hline Intravenous STS & 89 & $58(65.2 \%)$ & 70 & $45(64.3 \%)$ & 19 & $13(68.4 \%)$ \\
\hline STS cumulative dose $(\mathrm{g})$ & 56 & $488(300-750)$ & 43 & $525(300-750)$ & 13 & $375(225-900)$ \\
\hline STS duration (week) & 58 & $6(4-10)$ & 45 & $6(4-10)$ & 13 & $5(3-12)$ \\
\hline Renal Replacement Therapy modification & 89 & $57(64.0 \%)$ & & & & \\
\hline Increase of dialysis duration and/or frequency & & & 70 & $41(58.6 \%)$ & & \\
\hline Start of dialysis & & & & & 19 & $16(84.2 \%)$ \\
\hline Switch from HD to HDF & & & 70 & $13(18.6 \%)$ & & \\
\hline Switch from HDF to HD & & & 70 & $4(5.7 \%)$ & & \\
\hline Use of citrate dialysate & 89 & $6(6.7 \%)$ & 70 & $6(8.6 \%)$ & 19 & $0(0.0 \%)$ \\
\hline Discontinuation or lowering of oral calcium supply & 53 & $31(58.5 \%)$ & 46 & $26(56.5 \%)$ & 7 & $5(71.43 \%)$ \\
\hline Nutritional support therapy & 89 & $47(52.8 \%)$ & 70 & $35(50.0 \%)$ & 19 & $12(63.2 \%)$ \\
\hline Sevelamer & 89 & $42(47.2 \%)$ & 70 & $31(44.3 \%)$ & 19 & $11(57.9 \%)$ \\
\hline Initiation or dose increase of Sevelamer & 89 & $24(27.0 \%)$ & 70 & $15(21.4 \%)$ & 19 & $9(47.4 \%)$ \\
\hline Initiation or dose increase of Cinacalcet & 89 & $31(34.8 \%)$ & 70 & $27(38.6 \%)$ & 19 & $4(21.1 \%)$ \\
\hline Discontinuation of native vitamin $\mathrm{D}$ & 52 & $18(34.6 \%)$ & 40 & $12(30.0 \%)$ & 12 & $6(50.0 \%)$ \\
\hline Surgical debridement & 89 & $22(24.7 \%)$ & 70 & $17(24.3 \%)$ & 19 & $5(26.3 \%)$ \\
\hline$\geq 2$ surgical debridement & 89 & $9(10.1 \%)$ & 70 & $8(11.4 \%)$ & 19 & $1(5.26 \%)$ \\
\hline Amputation & 89 & $15(16.9 \%)$ & 70 & $13(18.6 \%)$ & 19 & $2(10.5 \%)$ \\
\hline Lanthanum carbonate & 89 & $15(16.9 \%)$ & 70 & $15(21.4 \%)$ & 19 & $0(0.0 \%)$ \\
\hline Initiation or dose increase of Lanthanum carbonate & 89 & $7(7.9 \%)$ & 70 & $7(10.0 \%)$ & 19 & $0(0.0 \%)$ \\
\hline Negative pressure wound therapy & 89 & $12(13.5 \%)$ & 70 & $8(11.4 \%)$ & 19 & $4(21.1 \%)$ \\
\hline Discontinuation of iron therapy & 62 & $8(12.9 \%)$ & 54 & $7(13.0 \%)$ & 8 & $1(12.5 \%)$ \\
\hline Standard oxygen therapy & 89 & $11(12.4 \%)$ & 70 & $9(12.9 \%)$ & 19 & $2(10.5 \%)$ \\
\hline Initiation or dose increase of statin & 89 & $10(11.2 \%)$ & 70 & $8(11.4 \%)$ & 19 & $2(10.5 \%)$ \\
\hline Skin transplantation & 89 & $8(9.0 \%)$ & 70 & $7(10.0 \%)$ & 19 & $1(5.3 \%)$ \\
\hline Parathyroidectomy & 89 & $5(5.6 \%)$ & 70 & $5(7.1 \%)$ & 19 & $0(0.0 \%)$ \\
\hline \multicolumn{7}{|l|}{ Steroids } \\
\hline Discontinuation or dose decrease of steroids & 12 & $7(58.3 \%)$ & 10 & $6(60.0 \%)$ & 2 & $1(50.0 \%)$ \\
\hline Initiation or dose increase of steroids & 12 & $2(16.7 \%)$ & 10 & $2(20.0 \%)$ & 0 & $0(0.0 \%)$ \\
\hline Local steroids treatement & 89 & $12(13.5 \%)$ & 70 & $9(12.9 \%)$ & 19 & $3(15.8 \%)$ \\
\hline
\end{tabular}


Table 5 Multivariate logistic regression analysis of risk factors of CUA in dialysis cases compared to matched dialysis controls

\begin{tabular}{lll}
\hline Parameter & OR $(95 \% \mathrm{Cl})$ & $p$-value \\
\hline $\begin{array}{l}\text { Body Mass Index, per } \\
5 \mathrm{~kg} / \mathrm{m}^{2} \text { increase }\end{array}$ & $1.56(1.08-2.27)$ & 0.02 \\
$\begin{array}{l}\text { Loss of weight within } \\
6 \text { months before } \\
\text { diagnosis, per } 1 \mathrm{~kg} \text { increment }\end{array}$ & $1.66(1.22-2.26)$ & 0.001 \\
$\begin{array}{l}\text { Coronary artery disease } \\
\text { Albumin variation between } \\
\text { diagnosis and } 6 \text { months before, } \\
\text { per } 5 \mathrm{~g} / \mathrm{L} \text { increment }\end{array}$ & $5.52(1.07-28.65)$ & 0.04 \\
$\begin{array}{l}\text { Serum phosphate } \\
\text { (worst value within } 6\end{array}$ & $0.19(0.05-0.70)$ & 0.01 \\
months before onset of & & \\
CUA), per 1 mmol/L & & \\
increment & $9.27(1.70-50.68)$ & 0.01 \\
Vitamin K Antagonist & & \\
\hline
\end{tabular}

hyperbaric oxygen (2.3\%), bisphosphonate (2.3\%) and vitamin $\mathrm{K}$ supplementation (1.1\%).

\section{CUA risk factors among dialyzed patients}

Univariate logistic regression analysis (Table S2) revealed dialyzed CUA patients had significantly more diabetes mellitus (Odds Ratio (OR) 2.7), diabetes and/or hypertension associated nephropathy (OR 3.0), symptomatic peripheral vascular disease (OR 2.0), history of cardiac failure (OR 4.6) or of pathologic fracture (OR 2.4). They had increased adjusted serum calcium (OR 9.2), serum phosphate (OR 4.6), calcium phosphate product (OR 2.0), normalized iPTH (OR 1.1) and CRP (OR 1.4) in the
6 months prior to identification of lesions (respectively OR 2.2; 5.4; 2.0; 1.1 and 1.1). Insulin (OR 2.1) and Erythropoiesis-Stimulating Agent (OR 2.6) also increased this risk. On the contrary, dialysis dose (eKt/V) (OR 0.2) and hemoglobin level at onset of lesions (OR 0.7 ) were associated with lower odds.

By multivariate analysis (Table 5), risk factors independently associated CUA in dialyzed patients were obesity, coronary artery disease, weight loss over the last 6 months, serum phosphate increase within 6 months before diagnosis and VKA therapy. As lower odds were associated with serum albumin increase within the 6 months before onset of lesions (OR 0.2), serum albumin decrease was also a risk factor of CUA among the dialysis cases.

\section{CUA outcome}

$40.4 \%$ of deaths due to calciphylaxis occurred during the first year after diagnosis and $56.2 \%$ after 5 years (Table 6). Mortality of dialysis cases was significantly higher than paired hemodialysis controls (Hazard Ratio (HR) 3.4; 95\% Confidence Interval (CI) 2.2-5.2; $p<0.001$ ) (Fig. 2). The median delay between the onset of lesions and death was 4.1 months (IQR 2.2-14.2). The main circumstance of death was in palliative care for CUA patients (49.1\%).

Complete healing of CUA lesions occurred in $37.1 \%$ (Table 6). The median delay between healing and diagnosis was 6.4 months (IQR 3.7-8.9). The median followup was respectively 6.1 (IQR $3.0-11.4$ ) and 16.1 (IQR 8.7-36.1) months in dialysis cases and controls.

Table 6 Evolution and outcome of CUA patients, compared to dialysis controls

\begin{tabular}{lllll}
\hline & Total CUA cases $(N=89)$ & Non dialysis cases $(N=19)$ & Dialysis cases $(N=70)$ & Dialysis controls $(N=140)$ \\
\hline Local evolution of CUA lesions & & & \\
Deterioration & $37(41.6 \%)$ & $7(36.4 \%)$ & $30(42.9 \%)$ \\
Any improvement & $2(2.3 \%)$ & $0(0.0 \%)$ & $2(2.9 \%)$ \\
Partial improvement & $17(19.9 \%)$ & $5(26.3 \%)$ & $12(17.1 \%)$ \\
Complete healing & $33(37.1 \%)$ & $7(36.8 \%)$ & $26(37.1 \%)$ & $14(36.8 \%)$ \\
Reccurence of CUA & $16(31.4 \%)$ & $2(15.4 \%)$ & & $18(12.9 \%)$ \\
Crude mortality rate (uncensored) & & & $28(40.0 \%)$ & $25(18.9 \%)$ \\
At 1 year after onset of lesions & $36(40.4 \%)$ & $8(42.1 \%)$ & $36(51.4 \%)$ & $44(31.4 \%)$ \\
At 2 years after onset of lesions & $46(51.7 \%)$ & $10(52.6 \%)$ & $40(57.1 \%)$ & $31(49.2 \%)$ \\
At 5years after onset of lesions & $50(56.2 \%)$ & $10(52.6 \%)$ & & $2(3.2 \%)$ \\
Cause of death & & & $8(18.6 \%)$ & $11(17.5 \%)$ \\
Cardiac arrest & $9(17.0 \%)$ & $1(10.0 \%)$ & $19(44.2 \%)$ & $3(4.8 \%)$ \\
Sepsis & $9(17.0 \%)$ & $1(10.0 \%)$ & $0(0.0 \%)$ & $0(0.0 \%)$ \\
Palliative care & $26(49.1 \%)$ & $7(70.0 \%)$ & $6(11.3 \%)$ & $0(0.0 \%)$ \\
Stroke & $0(0.0 \%)$ & $0(0.0 \%)$ & $31(72.1 \%)$ & \\
Cardiac failure & $6(11.3 \%)$ & $0(0.0 \%)$ & & \\
Death secondary to CUA & $38(71.7 \%)$ & $7(70.0 \%)$ & & \\
\hline
\end{tabular}



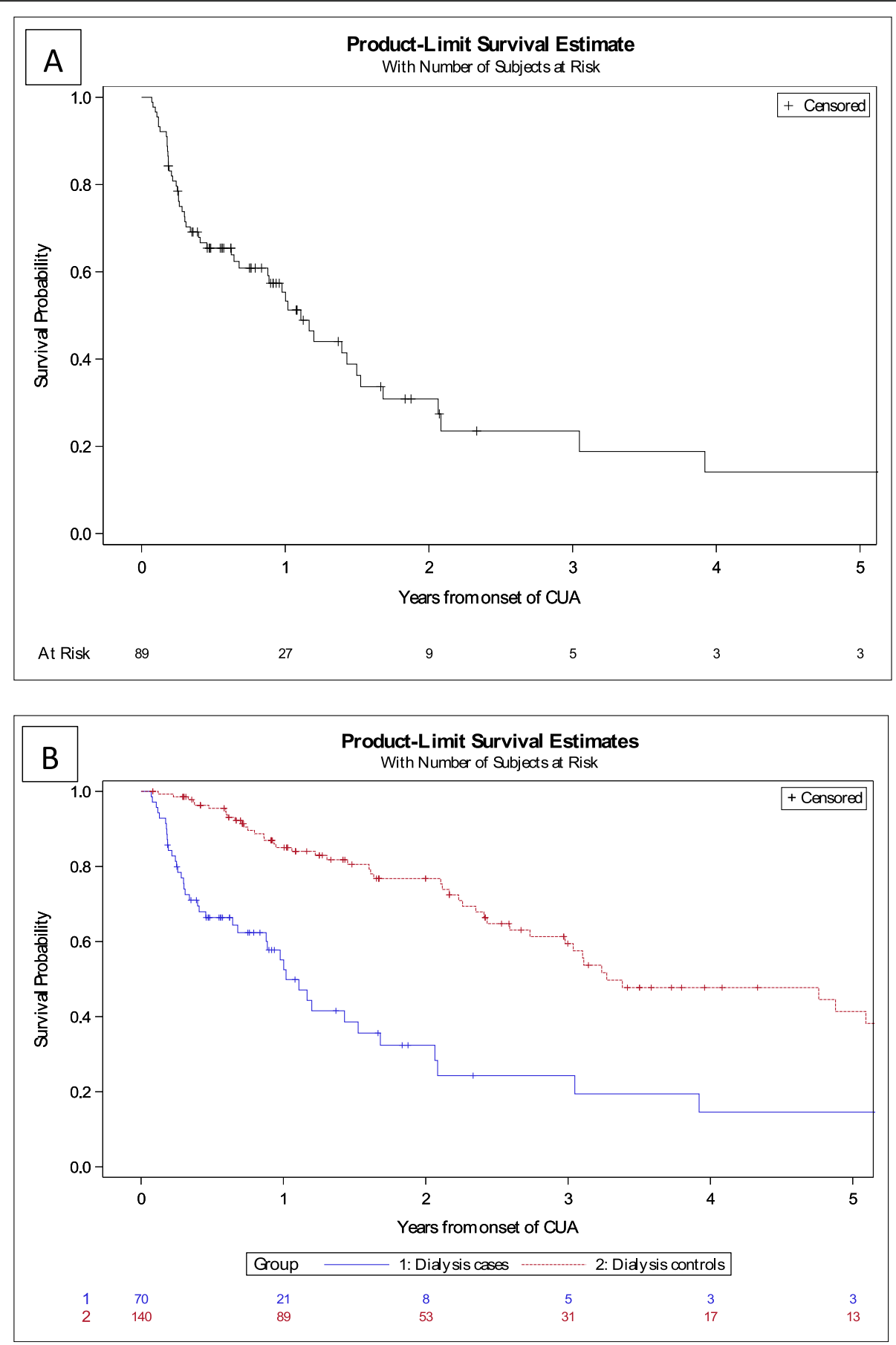

Fig. 2 Survival among Calcific Uremic Arteriolopathy cases and the hemodialysis controls

\section{Factors predictive of survival}

Higher BMI (HR 0.79; $p=0.004$ ), higher serum albumin at onset of lesions (HR $0.70 ; p<0.001)$ and VKA discontinuation (HR $0.41 ; p=0.01$ ) were associated with better survival in univariate analysis only (Table S3). After removal of patients treated less than 2 weeks or with a cumulative dose below $150 \mathrm{~g}$ of STS, a trend to better survival was associated with STS cumulated dose and duration (HR 0.87; CI 0.77-0.97; $p=0.02)$.

By multivariate analysis, items with $p<0.2$ in the univariate analysis were included, except sevelamer and lanthanum carbonate treatment at diagnosis, normalized $\mathrm{PTH}$, eKt/V, number of skin biopsies, STS duration, VKA discontinuation and CUA recurrence, because of missing data. Adjusted serum calcium and calcium phosphate 
product were removed because of linkage to serum calcium and phosphate. Thus 192 patients were included in the multivariate analysis. Finally, factors independently associated with survival were surgical debridement (HR 0.11 ) and antibiotherapy (HR 0.25) (Table 7), whereas parathyroidectomy increased the risk of death (HR 29.5).

\section{Discussion}

The 89 CUA patients of our study were typically 70-year old overweight diabetic females under VKA therapy (72\%). In these cases, bone mineral disease parameters were out of the recommended ranges and were associated with inflammation and malnutrition.

These patients were identified from all over western France and the informations provided by our study are the results of average clinical management of these patients and not the experience of one expert care center. By following the Hayashi criteria [8], a skin biopsy was not a

Table 7 Multivariate conditional logistic regression analysis of survival predictors among the 89 Calcific Uremic Arteriolopathy cases

\begin{tabular}{|c|c|c|}
\hline Parameter & $\mathrm{HR}(95 \% \mathrm{Cl})$ & $p$-value \\
\hline $\begin{array}{l}\text { Hemoglobin at diagnosis, } \\
\text { per } 1 \mathrm{~g} / \mathrm{dL} \text { increment }\end{array}$ & $0.42(0.30-0.60)$ & $<0.001$ \\
\hline Insulin therapy & $0.28(0.12-0.65)$ & 0.003 \\
\hline Lower limbs localization & & $<0.001$ \\
\hline Below knee VS none & $0.13(0.01-1.23)$ & 0.20 \\
\hline Above knee VS none & $0.17(0.01-2.49)$ & 0.72 \\
\hline Any localization VS none & $107.04(16.1-713)$ & $<0.001$ \\
\hline Upper limbs localization & & $<0.001$ \\
\hline Below elbow VS none & $10.79(2.34-49.7)$ & 0.002 \\
\hline Any localization VS none & $267.48(23.3-3069)$ & $<0.001$ \\
\hline Type of CUA & & $<0.001$ \\
\hline $\begin{array}{l}\text { Distal-type VS } \\
\text { proximal-type }\end{array}$ & $0.04(0.00-0.44)$ & 0.008 \\
\hline $\begin{array}{l}\text { Proximal and distal type } \\
\text { VS proximal type }\end{array}$ & $0.01(0.00-0.13)$ & $<0.001$ \\
\hline Parathyroidectomy & $29.53(3.87-226)$ & 0.001 \\
\hline Sevelamer & $0.26(0.10-0.66)$ & 0.005 \\
\hline Lanthanum carbonate & $0.04(0.01-0.21)$ & $<0.001$ \\
\hline Surgical debridement & $0.11(0.04-0.28)$ & $<0.001$ \\
\hline Antibiotherapy & $0.25(0.08-0.73)$ & 0.01 \\
\hline $\begin{array}{l}\text { Local evolution of } \\
\text { skin lesions }\end{array}$ & & $<0.001$ \\
\hline $\begin{array}{l}\text { Partial improvement } \\
\text { VS complete healing }\end{array}$ & $1.14(0.22-5.77)$ & 0.88 \\
\hline $\begin{array}{l}\text { Deterioration VS } \\
\text { complete healing }\end{array}$ & $497.78(79.5-3118)$ & $<0.001$ \\
\hline $\begin{array}{l}\text { No improvement } \\
\text { VS complete healing }\end{array}$ & $112.48(12.4-1023)$ & $<0.001$ \\
\hline
\end{tabular}

Hazard ratio (HR) with $95 \%$ confidence interval prerequisite in our study and confirmed diagnosis only in required clinical situations, in particular, to rule out a differential diagnosis. Because this can worsen lesions, skin biopsies are frequently avoided. Specificity is also questioned because of the frequence of extravascular calcifications in ESRD. Skin biopsies can confirm diagnosis by showing the combination of arteriolar media calcification and thrombosis that is associated with CUA [9]. We think that the identified CUA cases are the reflect of complexity of CUA diagnosis in clinical practice.

The demographic data and CUA predisposing factors identified in our study were consistent with other casecontrol studies $[4,10]$. Inflammation and bone mineral disease abnormalities, especially hyperphosphatemia and hyperparathyroidism, and malnutrition preceded CUA onset by months $[2,10]$. For the first time, weight loss within the 6 months before CUA onset was identified by our study as a risk factor in dialysis patients.

The association of CUA and VKA therapy has already been reported $[4,8,11]$. By decreasing carboxylated matrix Gla Protein (cMGP), VKA are suspected to contribute to vascular calcification and therefore promote calciphylaxis. A low level of cMGP have also been highlighted in CUA cases associated with Vitamin K deficiency [12]. Besides, Warfarin could paradoxically favor thrombosis locally, by blocking protein $\mathrm{S}$ endothelial secretion in response to stress [13]. Thrombophilia is also a known risk factor of calciphylaxis $[14,15]$ and we assume that the low prevalence of thrombophilia in our study might be due to lack of systematic screening. As Direct oral anticoagulants have no pro-thrombotic effect and given that vessel thrombosis may play a key role in calciphylaxis, they have been used to replace VKA once diagnosis of CUA is confirmed [16, 17]. Two retrospective studies $[16,17]$ have assessed the safety of Apixaban in CUA patients: 4 bleeding events in 20 dialysis patients were found and a lower mortality rate was demonstrated. Additional comparative studies are of course necessary.

We were surprised to identify so many non-dialyzed patients with calciphylaxis. The frontier between uremic calciphylaxis and non-uremic calciphylaxis is difficult to define. Studies on non-uremic calciphylaxis are mainly case reports and case series. Interestingly, a review on Non Nephrogenic Calciphylaxis (NNC), defined as calciphylaxis occurring in patients without impaired renal function $\left(\mathrm{eGFR}>60 \mathrm{~mL} / \mathrm{min} / 1.73 \mathrm{~m}^{2}\right.$ ), showed that VKA and obesity were the two main conditions associated with NNC [18]. Mean blood mineral parameters were normal. Calciphylaxis seems to be the conjunction of multiple conditions (obesity, VKA, bone mineral disease abnormalities, uremia, inflammation) with a broad spectrum of variations. In our study, non-dialyzed CUA patients were more obese and inflammation prior CUA onset was more severe than in dialyzed CUA patients. A 
link between arteriolar calcification and adipocytes could explain the preferential localization of calciphylaxis in adipose tissue areas and the increased risk of calciphylaxis associated with obesity $[4,19]$. Childhood obesity is already known to increase coronary artery calcification by middle age [20]. An increase in NNC reported cases [18, 19] might not only be secondary to increased medical awareness of this disease, but also might be linked to the epidemic of obesity. In our study, non-dialyzed cases had the same survival as dialysis cases, whereas some studies have reported a better prognosis of NNC [21].

The mortality rate was particularly high in our study. UIcerative lesions and proximal-type CUA, known as poor prognosis factors $[2,3]$, were predominant. The prolonged diagnosis delay (46 vs 28 days in the German registry [22]) could be explained by the use of ulcerative skin lesions as inclusion criteria, because non-ulcerative lesions (plaques, nodules) precede the ulcerative lesions by several days [3]. This diagnosis delay could also be linked to a lack of acknowledgment of early CUA lesions, as seen in the Japanese case control study [10]. The dialysis vintage, shorter among the dialyzed cases than the matched controls ( 1.4 vs 2.2 years) could not explain the excess of mortality in dialyzed cases. So calciphylaxis does not necessarily occur after a long dialysis vintage. This is consistent with the important number of CUA cases identified among patients with CKD stage 4-5.

The main circumstance of death in our study was palliative care (49\%), in the absence of efficient therapy to treat CUA. Due to its promising treatment properties [23, 24], STS was frequently administered, but was only associated with a trend toward better prognosis after exclusion of patients treated less than 2 weeks. Trials are in progress to assess the real benefit of STS in CUA. As demonstrated by other studies, surgical debridement had a net impact on survival [25-27] and should be proposed more widely. Contrary to other studies [26], parathyroidectomy was associated with a poorer prognosis, but given that only 5 parathyroidectomies were performed the conclusions are limited.

\section{Conclusions}

Our study confirms the data reported by others on CUA but showed for the first time the contribution of significant unintentional weight loss. Few therapeutic measures seem efficient. Among them, STS is commonly used, but its benefit has still to be proved. The ongoing trials are of major interest.

\section{Supplementary information}

Supplementary information accompanies this paper at https://doi.org/10. 1186/s12882-020-01722-y.

Additional file 1: Table S1. Differential diagnosis idendified among eligible patients. Table S2. Univariate logistic regression analysis of risk factors of CUA in dialysis cases compared to matched dialysis controls. Table S3. Univariate conditional logistic regression analysis of survival predictors among Calcific Uremic Arteriolopathy cases.

\section{Abbreviations \\ BMI: Body Mass Index; CKD: Chronic Kidney Disease; CMGP: carboxylated Matrix Gla Protein; CRP: C-Reactive Protein; CUA: Calcific Uremic Arteriolopathy; eGFR: estimated Glomerular Filtration Rate; ESRD: End Stage Renal Disease; HR: Hazard Ratio; iPTH: intact Parathyroid Hormone; IQR: Interquartile Range; NNC: Non Nephrogenic Calciphylaxis; OR: Odds Ratio; STS: Sodium Thiosulfate; VKA: Vitamin K antagonist}

\section{Acknowledgments}

The authors would like to thank the following nephrologists for reporting cases of CUA and allowing to collect data on dialyzed controls in their care center: Dr. F. Babinet (Centre Echo Le Mans), Dr. C. Bachelet-Rousseau (Centre Hospitalier Saint Louis, La Rochelle), Dr. S. Batula (AUB Saint Brieuc), Dr. D. Besnier (Centre Hospitalier Saint Nazaire), Dr. V. Besson (CHU Angers), Dr. H. Bonarek (Centre Hospitalier de Saintes), Dr. C. Charasse (Centre Hospitalier Saint Brieuc), Dr. K. El Nasser (Centre Hospitalier de Cholet), Dr. M. François (CHU Tours), Dr. A. Ganea (Centre Hospitalier d'Orléans), Dr. R. Gautard (Clinique Saint Gatien, Tours), Dr. M. P. Guillodo (AUB Brest), Dr. D. Labatut (Centre

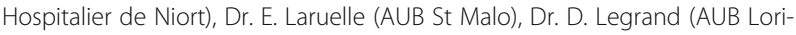
ent), Dr. A. Le Guillou (Centre Hospitalier de Lorient), Dr. E. Maksour (Centre Hospitalier de Bourges), Dr. L. Mandart (Centre Hospitalier de Vannes), Dr. G. Piccoli (Centre Hospitalier Le Mans), Dr. L.M. Pouteau (Centre Hospitalier de Laval), Dr. G. Queffeulou (Centre Hospitalier de Cherbourg), Dr. E. Renaudineau (Centre Hospitalier de St Malo), Dr. N. Target (Centre Hospitalier Départemental, La Roche sur Yon), Dr. A. Testa (Centre Echo Nantes), Pr C. Vigneau (CHU Rennes) and Dr. E. Zagdoun (Centre Hospitalier Mémorial de Saint Lô). The authors would like to acknowledge also research coordinators of REIN registry (Claudette Berquez, Assia Hami, Fabien Duthe, Muriel Siebert) for their help.

\section{Authors' contributions}

$\mathrm{RG}$ and $\mathrm{DL}$ designed the study, recruited the cases and the controls, collected, analyzed and interpreted the data and wrote the manuscript. MP performed the statistical analysis. VM selected the CUA patients from the $\mathrm{ECHO}$ database. $\mathrm{MH}$ contributed to the writing and revision of the manuscript. All authors read and approved the final manuscript.

Funding

No funding

\section{Availability of data and materials}

All data generated or analysed during this study are included in this published article and its supplementary information files.

\section{Ethics approval and consent to participate}

The study was approved by the ethics committee of the Nantes University Hospital (Groupe Nantais d'Etudes dans le Domaine de la Santé (GNEDS)) on 20th January 2016. A written consent form was given to each patient, except for deceased patients and loss of follow-up patients.

\section{Consent for publication}

Not applicable.

\section{Competing interests}

VM reports receiving grant support from Meditor and consulting fees from Astellas. The other authors declare that they have no competing interests.

\section{Author details}

'Department of Nephrology and Immunology, Institute of Transplantation Urology and Nephrology, Centre Hospitalier Universitaire de Nantes, Nantes, France. ${ }^{2}$ Service de Néphrologie et Immunologie Clinique, Centre Hospitalier Universitaire de Nantes, 30, bd Jean Monnet 44093, Nantes Cedex 01, France. ${ }^{3}$ Biostatistician, Direction de la Recherche, Plateforme de Méthodologie et Biostatistiques, Centre Hospitalier Universitaire de Nantes, Nantes, France. ${ }^{4} \mathrm{ECHO}$ Department of Medical Information, Nantes, France. ${ }^{5}$ Department of Nephrology, Centre Hospitalier de Saint Nazaire, St Nazaire, France. 
Received: 27 September 2019 Accepted: 11 February 2020

Published online: 26 February 2020

\section{References}

1. Nigwekar SU, Kroshinksy D, Nazarian RM, et al. Calciphylaxis: risk factors, diagnosis, and treatment. Am J Kidney Dis Off J Natl Kidney Found. 2015; 66(1):133-46.

2. Mazhar AR, Johnson RJ, Gillen D, et al. Risk factors and mortality associated with calciphylaxis in end-stage renal disease. Kidney Int. 2001;60(1):324-32.

3. Fine A, Zacharias J. Calciphylaxis is usually non-ulcerating: risk factors, outcome and therapy. Kidney Int. 2002;61(6):2210-7.

4. Nigwekar SU, Zhao S, Wenger J, et al. A nationally representative study of calcific uremic Arteriolopathy risk factors. J Am Soc Nephrol. 2016;27(11): 3421-9.

5. Brandenburg VM, Evenepoel P, Floege J, et al. Lack of evidence does not justify neglect: how can we address unmet medical needs in calciphylaxis? Nephrol Dial Transplant. 2016;31(8):1211-9.

6. Nigwekar SU, Thadhani R, Brandenburg VM. Calciphylaxis. N Engl J Med. 2018;378(18):1704-14.

7. Chen TY, Lehman JS, Gibson LE, et al. Histopathology of Calciphylaxis: cohort study with clinical correlations. Am J Dermatopathol. 2017;39(11):795.

8. Hayashi M. Calciphylaxis: diagnosis and clinical features. Clin Exp Nephrol. 2013;17(4):498-503.

9. Ellis $C L, O^{\prime} N e i l l ~ W C$. Questionable specificity of histologic findings in calcific uremic arteriolopathy. Kidney Int. 2018;94(2):390-5.

10. Hayashi M, Takamatsu I, Kanno Y, et al. A case-control study of calciphylaxis in Japanese end-stage renal disease patients. Nephrol Dial Transplant. 2012; 27(4):1580-4.

11. Nigwekar SU, Bhan I, Turchin A, et al. Statin use and calcific uremic Arteriolopathy: a matched case-control study. Am J Nephrol. 2013;37(4): 325-32.

12. Nigwekar SU, Bloch DB, Nazarian RM, et al. Vitamin K-dependent carboxylation of matrix Gla protein influences the risk of Calciphylaxis. J Am Soc Nephrol. 2017;28(6):1717-22.

13. Stern D. Participation of endothelial cells in the protein C-protein S anticoagulant pathway: the synthesis and release of protein S. J Cell Biol. 1986;102(5):1971-8.

14. El-Azhary RA, Patzelt MT, McBane RD, et al. Calciphylaxis: a disease of Pannicular thrombosis. Mayo Clin Proc. 2016;91(10):1395-402.

15. Dobry AS, Ko LN, St John J, et al. Association between Hypercoagulable conditions and Calciphylaxis in patients with renal disease: a case-control study. JAMA Dermatol. 2018;154(2):182.

16. King BJ, El-Azhary RA, McEvoy MT, et al. Direct oral anticoagulant medications in calciphylaxis. Int J Dermatol. 2017;56(10):1065-70.

17. Garza-Mayers AC, Shah R, Sykes DB, et al. The successful use of Apixaban in Dialysis patients with Calciphylaxis who require anticoagulation: a retrospective analysis. Am J Nephrol. 2018;48(3):168-71.

18. Bajaj R, Courbebaisse M, Kroshinsky D, et al. Calciphylaxis in patients with Normal renal function: a case series and systematic review. Mayo Clin Proc. 2018:93(9):1202-12.

19. Nigwekar SU, Wolf M, Sterns RH, et al. Calciphylaxis from nonuremic causes: a systematic review. Clin J Am Soc Nephrol. 2008;3(4):1139-43.

20. Reis JP, Loria CM, Lewis CE, et al. Association between duration of overall and abdominal obesity beginning in young adulthood and coronary artery calcification in middle age. JAMA. 2013;310(3):280-8.

21. Yu WY-H, Bhutani T, Kornik R, et al. Warfarin-associated nonuremic Calciphylaxis. JAMA Dermatol. 2017;153(3):309-14.

22. Brandenburg VM, Kramann $\mathrm{R}$, Rothe $\mathrm{H}$, et al. Calcific uraemic arteriolopathy (calciphylaxis): data from a large nationwide registry. Nephrol Dial Transplant. 2017;32(1):126-32.

23. Nigwekar SU, Brunelli SM, Meade D, et al. Sodium thiosulfate therapy for calcific uremic Arteriolopathy. Clin J Am Soc Nephrol. 2013;8(7):1162-70.

24. Zitt E, König M, Vychytil A, et al. Use of sodium thiosulphate in a multiinterventional setting for the treatment of calciphylaxis in dialysis patients. Nephrol Dial Transplant. 2013;28(5):1232-40.

25. Weenig RH, Sewell LD, Davis MDP, et al. Calciphylaxis: natural history, risk factor analysis, and outcome. J Am Acad Dermatol. 2007:56(4):569-79.

26. McCarthy JT, El-Azhary RA, Patzelt MT, et al. Survival, risk factors, and effect of treatment in 101 patients with Calciphylaxis. Mayo Clin Proc. 2016;91(10): 1384-94.
27. Lal G, Nowell AG, Liao J, et al. Determinants of survival in patients with calciphylaxis: a multivariate analysis. Surgery. 2009;146(6):1028-34.

\section{Publisher's Note}

Springer Nature remains neutral with regard to jurisdictional claims in published maps and institutional affiliations.

\section{Ready to submit your research? Choose BMC and benefit from:}

- fast, convenient online submission

- thorough peer review by experienced researchers in your field

- rapid publication on acceptance

- support for research data, including large and complex data types

- gold Open Access which fosters wider collaboration and increased citations

- maximum visibility for your research: over $100 \mathrm{M}$ website views per year

At BMC, research is always in progress.

Learn more biomedcentral.com/submissions 